\title{
Desidratação osmótica para obtenção de cagaita passa
}

Caroline Dantas Mendes da SILVA ${ }^{[1]}$, Caroline Roberta Freitas PIRES ${ }^{[1]}$, Juliana Pinto LIMA ${ }^{[1]}$, Anielli Souza PEREIRA $^{[2]}$ e Clemilson Antônio SILVA ${ }^{[1]}$

${ }^{[1]}$ Universidade Federal do Tocantins (UFT), Campus Palmas. Avenida NS 15, 109 Norte - Plano Diretor Norte, 77001-090. Palmas-TO, Brasil. Email: carol_dms@uft.edu.br; carolinerfpires@uft.edu.br; jujuufv@yahoo.com.br; clemilsonsilva@uft.edu.br

${ }^{[2]}$ Universidade Federal de Lavras (UFLA). Departamento de Microbiologia agrícola. Caixa Postal 3037, 37200-000. Lavras-MG, Brasil. E-mail:anisouza21@hotmail.com

INFORMAÇÕES RESUMO

Recebido em: 05/11/2015

Aceito em: 30/11/2015

Publicado em: 23/12/2015

A cagaita é um fruto do cerrado globoso, de coloração amarelo-clara e contendo de 1 a 3 sementes brancas, envoltas em uma polpa levemente ácida. Apresenta um conteúdo apreciável de vitamina $\mathrm{C}$, fibras e de carotenoides. O presente trabalho foi realizado com o objetivo de avaliar as condições de desidratação osmótica para a obtenção de

Document Object Identifier

$10.18067 /$ jbfs.v2i4.82 passas de cagaita. $\mathrm{O}$ tratamento osmótico foi conduzido a $50^{\circ} \mathrm{C}$ e a $70^{\circ} \mathrm{C}$, utilizando-se de soluções de sacarose a $45^{\circ}$ Brix e a $65^{\circ}$ Brix. Avaliaram-se os parâmetros perda de

Termos de indexação: água, perda de massa e incorporação de sólidos, taxa de encolhimento e capacidade de rehidratação. As amostras desidratadas foram submetidas à avaliação sensorial

Eugenia dysenterica

Qualidade aplicando-se um teste de aceitação. O estudo mostrou que houve maior perda de água, perda de massa e maior incorporação de sólidos solúveis nas amostras de cagaita desidratadas nas condições de $65^{\circ}$ Brix a $70^{\circ} \mathrm{C}$. A menor taxa de encolhimento e a

Desidratação osmótica

*Autor para correspondência maior capacidade de rehidratação foram observadas nas amostras desidratadas osmoticamente na solução de $45^{\circ}$ Brix a $50^{\circ} \mathrm{C}$. A análise sensorial evidenciou que a cagaita desidratada apresentou boa aceitação, constituindo uma opção válida de carol_dms@uft.edu.br agregação de valor ao fruto.

\section{Osmotic dehydration to obtain cagaita raisins}

\begin{abstract}
The cagaita is a fruit of cerrado, of pale yellow color and containing 1-3 white seeds, wrapped in a slightly acidic pulp. It presents a significant content of vitamin $\mathrm{C}$, carotenoids and fibers. This work was carried out to evaluate the osmotic dehydration conditions for obtaining cagaita raisins. The osmotic treatment was conducted at $50{ }^{\circ} \mathrm{C}$ and $70{ }^{\circ} \mathrm{C}$, without stirring, using sucrose solutions at $45^{\circ}$ Brix and $65{ }^{\circ}$ Brix. Evaluated the loss of water parameters, weight loss and solids uptake, shrinkage and rehydration capacity. The dehydrated samples were subjected to sensory evaluation by applying an acceptance test. The study showed that there was a greater water loss, weight loss and increased incorporation of soluble solids in the dried samples cagaita under the conditions of 65 ${ }^{\circ}$ Brix to $70{ }^{\circ} \mathrm{C}$. The lower shrinkage rate and higher rehydration capacity were observed in osmotically dehydrated samples at $45^{\circ}$ Brix solution at $50{ }^{\circ} \mathrm{C}$. Sensory analysis showed that dehydrated cagaita showed good acceptance, constituting an option to add value to the fruit.
\end{abstract}

Index terms: Campomanesia pubescens; quality; osmotic dehydration

(1) Copyright: (C) 2015 JBFS all rights. This is an open-access article distributed under the terms of the Creative Commons Attribution License, which permits unrestricted use, distribution, and reproduction in any medium, provided the original author and source are credited.

Financiamento: Os autores reportam que não houve suporte e auxílio financeiro.

Conflito de interesse: Os autores declaram que não há conflito de interesse.

Como referir esse documento (ABNT):

SILVA, C. D. M.; PIRES, C. R. F.; LIMA, J. P.; PEREIRA, A. S.; SILVA, C. A. Desidratação osmótica para obtenção de cagaita passa. Journal of Bioenergy and Food Science, Macapá, v.2, n.4, p.226-233, out./dez., 2015 http://dx.doi.org/10.18067/jbfs.v2i4.82

\section{INTRODUÇÃO}

O bioma Cerrado se destaca por apresentar uma elevada biodiversidade, com frutíferas nativas que são vendidas em feiras da região e utilizadas na culinária, além de sua utilização pelos setores farmacêutico e também na extração de óleos pela indústria oleoquímica. No entanto, no cenário atual tem-se observado uma substituição da vegetação do cerrado por monoculturas e pastagens para criação de bovinos [1].

Uma das espécies nativa do cerrado que pode ser aproveitada na indústria de alimentos é 
conhecida popularmente como cagaita (Eugenia dysenterica), caracterizada por frutos globosos, de coloração amarelo-clara, contendo de 1 a 3 sementes brancas, envoltas em uma polpa levemente ácida. Apresenta um conteúdo apreciável de vitamina $\mathrm{C}$, fibras e de carotenoides [2].

Atualmente tem-se observado uma
modificação nos hábitos alimentares dos
consumidores, repercutindo em maior estímulo ao
consumo de frutas e hortaliças, tanto in natura
como processadas [3].

O aproveitamento alimentar de algumas espécies nativas do cerrado como o buriti, murici e o pequi já foram relatados na literatura [4-6].

Uma das alternativas viáveis para a agregação de valor aos frutos e prolongamento de sua vida útil, consiste no processo de secagem onde destaca-se a desidratação osmótica que ocasiona a eliminação da água, aumentando a vida de prateleira do produto pela redução das alterações físicoquímicas e biológicas [7]. No processo de desidratação osmótico ocorre a passagem da água do interior do fruto para a solução concentrada e do soluto para o interior do fruto [8].

No Brasil, a desidratação osmótica de frutas tem sido considerada uma tecnologia promissora, visto que, apresenta uma elevada diversidade de frutos, além da grande produtividade de sacarose oriunda da cana-de-açúcar, sendo este um soluto que ocasiona pequenas modificações no decorrer do processo $[9,10]$.

Portanto, a desidratação osmótica utilizada como método alternativo para a obtenção de frutas passa favorece na obtenção de produtos com textura, cor e sabor adequados [10].

Diante disso, o objetivo deste trabalho foi avaliar o efeito das variáveis temperatura e concentração do xarope de sacarose nos parâmetros de desidratação osmótica, características físicoquímicas e qualidade sensorial dos frutos de cagaita desidratados.

\section{MATERIAL E MÉTODOS}

Os frutos cagaita foram colhidos no estádio de maturação comercial no município de Palmas TO. Os frutos foram selecionados quanto à ausência de injúrias e com coloração uniforme. Em seguida foram lavados em água corrente visando a remoção de sujidades maiores e sanificados com solução de hipoclorito de sódio a $200 \mathrm{ppm}$ por um período de 15 minutos com posterior enxágue. Após a etapa de sanitização, os frutos foram submetidos ao branqueamento e sequencialmente imersos em uma solução de ácido ascórbico, proporção 1:3 (fruto:solução), na concentração de 300ppm por seis minutos. Para efetuar a desidratação osmótica utilizou-se a sacarose.

Durante a desidratação osmótica os frutos foram acondicionados em béqueres de 1 litro com as soluções osmóticas nas concentrações de 45 e $65^{\circ}$ Brix. Os frutos permaneceram em contato com a solução osmótica por 8 horas à temperatura de 50 e $70^{\circ} \mathrm{C}$. A proporção fruta/solução adotada foi de 1:5 $(\mathrm{p} / \mathrm{p})$. Após a desidratação os frutos foram depositados sobre um papel absorvente visando retirar o excedente da solução de sacarose. Em seguida os frutos foram conduzidos para estufa à temperatura de $65^{\circ} \mathrm{C}$ por aproximadamente 6 horas, para a remoção do teor de umidade até alcançar um valor entre 20 a $22 \%$ (base úmida).

Após a desidratação os frutos foram armazenados em sacos de polietileno, visando preservar as características sensoriais até $o$ momento das análises que foram conduzidas após 48 horas. Na Tabela 1 estão apresentados os tratamentos osmóticos que os frutos foram submetidos.

Tabela 1. Tratamentos utilizados na desidratação osmótica da cagaita.

\begin{tabular}{cl}
\hline Codificação & \multicolumn{1}{c}{ Tratamentos } \\
\hline $\mathrm{T} 1$ & Xarope de sacarose a $45^{\circ}$ Brix (proporção fruto:xarope 1:5) à $50^{\circ} \mathrm{C}$. \\
$\mathrm{T} 2$ & Xarope de sacarose a $45^{\circ} \mathrm{Brix}$ (proporção fruto:xarope 1:5) à $70^{\circ} \mathrm{C}$. \\
$\mathrm{T} 3$ & Xarope de sacarose a $65^{\circ}$ Brix (proporção fruto:xarope 1:5) à $50^{\circ} \mathrm{C}$. \\
$\mathrm{T} 4$ & Xarope de sacarose a $65^{\circ}$ Brix (proporção fruto:xarope 1:5) à $70^{\circ} \mathrm{C}$ \\
\hline
\end{tabular}

A determinação do $\mathrm{pH}$ consistiu em pesar 5 gramas da amostra de cagaita-passa e solubilizar em $45 \mathrm{ml}$ de água, obtendo uma solução final de $50 \mathrm{ml}$. Em seguida foi feito a aferição em pHmetro digital AOAC [11]. A acidez total titulável (ATT) foi determinada por titulação com $\mathrm{NaOH} \quad 0,1 \mathrm{~N}$ e utilizando a fenolftaleína como indicador. Os resultados foram expressos em \% de ácido cítrico. Os sólidos solúveis totais (SST) foram expressos em ${ }^{\circ}$ Brix com a utilização de um refratômetro digital AOAC [11]. Após a determinação da amostra de cagaita-passa mais aceita pelos provadores, estas foram caracterizadas nutricionalmente. A determinação da umidade, 
proteína, lipídios, fibras e cinzas foram feitas seguindo a AOAC [11]. Para a determinação da fração glicídica $\left(\mathrm{g} 100 \mathrm{~g}^{-1}\right.$ da matéria integral) utilizou-se a equação: \% Fração glicídica $=100-(\%$ de umidade + de extrato etéreo $+\%$ de proteína bruta $+\%$ de fibra bruta $+\%$ fração cinzas), considerando a matéria integral. O valor de Kcal foi obtido a partir dos valores encontrados para proteína, lipídios e carboidratos disponíveis por diferença utilizando os fatores de conversão de Atwater. $\mathrm{Kcal}=(4 \mathrm{x} \mathrm{g}$ de carboidratos $)+(4 \mathrm{x} \mathrm{g}$ de proteínas $)+(9$ x g de lipídios) .

Para a determinação dos parâmetros incorporação de sólidos (IS), perda de massa (PM) e perda de água (PA), expressos por (g água $100 \mathrm{~g}^{-1}$ massa inicial), utilizou-se as equações abaixo:

$\mathrm{PA}=\left(\mathrm{U}_{\mathrm{f}} \mathrm{M}_{\mathrm{f}}-\mathrm{U}_{\mathrm{i}} \mathrm{M}_{\mathrm{i}}\right) / \mathrm{M}_{\mathrm{i}} \times 100$

$\mathrm{PM}=\left(\mathrm{M}_{\mathrm{f}}-\mathrm{M}_{\mathrm{i}}\right) / \mathrm{M}_{\mathrm{i}} \times 100$

$\mathrm{IS}=\left(\mathrm{ST}_{\mathrm{f}} \mathrm{M}_{\mathrm{f}}-\mathrm{ST}_{\mathrm{i}} \mathrm{M}_{\mathrm{i}} / \mathrm{M}_{\mathrm{i}}\right) \times 100$

Onde:

$\mathrm{U}_{\mathrm{f}}$ - corresponde ao teor de umidade no final do processo, \%;

$\mathrm{M}_{\mathrm{f}}$ - massa no final do processo, $\mathrm{g}$;

$\mathrm{U}_{\mathrm{i}}$ - teor de umidade inicial, \%;
$\mathrm{M}_{\mathrm{i}}$ - massa inicial, $\mathrm{g}$;

$\mathrm{ST}_{\mathrm{f}}$ - teor de sólidos totais no final do processo, \%;

$\mathrm{St}_{\mathrm{i}}$ - teor de sólidos iniciais, $\%$.

A determinação do encolhimento foi calculada através do deslocamento do tolueno em uma proveta descrito por Zogzas et al. [12], enquanto a capacidade de reidratação foi feita de acordo com o método proposto pela United States of Department Agriculture - USDA (1944), segundo a equação abaixo:

Água $_{\mathrm{abs}}(\%)=\left(\mathrm{M}_{\mathrm{f}}-\mathrm{M}_{\mathrm{o}}\right) / \mathrm{M}_{\mathrm{o}}$ × 100

Eq. (4)

Onde:

Águaabs-água absorvida (\%);

$\mathrm{M}_{\mathrm{o}^{-}}$massa inicial da amostra $(\mathrm{g})$; e

Mf-massa final da amostra após período de imersão (g).

As três amostras destinadas à avaliação sensorial foram obtidas a partir da avaliação da qualidade visual realizada pelo próprio observador e condutor do experimento através da atribuição de notas entre 1 (melhor qualidade) e 5 (menor qualidade) [13], de acordo com a Tabela 2.

Tabela 2. Índice de avaliação da qualidade visual de cagaita obtidas por desidratação osmótica.

\begin{tabular}{cll}
\hline Índice & Aparência & Descrição \\
\hline 1 & Excelente & Excelente aparência em geral, secagem uniforme sem alteração da cor \\
2 & Bom & Boa aparência em geral, secagem menos uniforme, ligeira alteração da cor \\
3 & Regular & Aparência regular, secagem desuniforme, escurecimento torna-se visível \\
4 & Ruim & Aparência ruim, secagem desuniforme, escurecimento intenso de algumas amostras \\
5 & Péssimo & Aparência péssima, secagem desuniforme, escurecimento intenso de algumas amostras. \\
\hline
\end{tabular}

Os testes de aceitação foram realizados na Universidade Federal do Tocantins, com 60 julgadores não treinados, homens e mulheres com idade entre 18 e 60 anos, consumidores de frutas secas. Para avaliar a aceitação com relação aos atributos cor, sabor, aroma e impressão global, foi utilizada uma escala hedônica estruturada de nove pontos $(1=$ desgostei extremamente; $5=$ nem gostei/nem desgostei; $9=$ gostei extremamente) [14]. Já a intenção de compra do produto foi avaliada utilizando uma escala hedônica de cinco pontos, onde $1=$ certamente não compraria e $5=$ certamente compraria. Os resultados obtidos foram submetidos à análise de variância (ANOVA) a as médias foram comparadas pelo teste de Tukey $(5 \%$ de significância), empregando-se o programa SISVAR [15].

\section{RESULTADOS E DISCUSSÃO}

A caracterização físico-química da cagaita in natura está apresentada na Tabela 3. Pelos resultados foi encontrado um valor de $92,8 \%$ de umidade, $0,78 \%$ de proteína, $0,37 \%$ de lipídios, $1,21 \%$ de fibra, $0,13 \%$ de cinzas e $4,71 \%$ de carboidratos. Silva [16] (2008) ao avaliar a composição química da cagaita (Eugenia dysenterica Dc) encontrou valores médios de proteínas de $0,82 \%$, lipídeos de $0,44 \%$, umidade de $94,34 \%$, fibra de $1,04 \%$, carboidratos de $3,08 \%$, e cinzas de $0,28 \%$. 
Tabela 3. Análise físico-química de cagaita in natura e na forma desidratada.

\begin{tabular}{lcc}
\hline $\begin{array}{c}\text { Características } \\
\text { físico-químicas }\end{array}$ & $\begin{array}{c}\text { Cagaita in } \\
\text { natura }\end{array}$ & $\begin{array}{c}\text { Cagaita Passa } \\
\left(45^{\circ} \text { Brix } / 50^{\circ} \mathrm{C}\right)\end{array}$ \\
\hline Umidade & $92,8 \pm 0,03$ & $21,75 \pm 0,08$ \\
Proteínas & $0,78 \pm 0,54$ & $1,67 \pm 0,35$ \\
Lipídios & $0,37 \pm 0,18$ & $0,82 \pm 0,54$ \\
Fibra & $1,21 \pm 0,33$ & $2,48 \pm 0,22$ \\
Cinzas & $0,13 \pm 0,06$ & $0,29 \pm 0,34$ \\
Carboidratos & $4,71 \pm 0,81$ & $72,99 \pm 0,21$ \\
Kcal & 25,29 & 306,02 \\
pH & $2,84 \pm 0,02$ & $2,63 \pm 0,02$ \\
Acidez & $6,34 \pm 0,07$ & $6,12 \pm 0,09$ \\
Sólidos & $7,3 \pm 0,10$ & $22,37 \pm 0,04$ \\
Solúveis & & \\
\hline
\end{tabular}

Os valores médios das variáveis incorporação de sólidos solúveis, perda de massa, perda de água, taxa de encolhimento e capacidade de reidratação das amostras de cagaita estão apresentados na Tabela 04.

Diversos fatores tais como concentração da solução de sacarose, temperatura da solução, além do tempo e proporção da fruta/ solução influenciam a taxa de perda de água no decorrer do processo de desidratação osmótica [16, 17].

No presente estudo foi adotado um tempo de 8 horas de desidratação osmótica mais 6 horas de estufa a $65^{\circ} \mathrm{C}$. No entanto, é importante destacar que o tempo necessário para a desidratação osmótica é variável para cada vegetal [18].

De acordo com a Tabela 04 é possível observar que as amostras de cagaita imersas em solução com $45^{\circ} \mathrm{Brix} / 50^{\circ} \mathrm{C}$ se diferiram das amostras imersas na solução de $45^{\circ} \mathrm{Brix} / 70^{\circ} \mathrm{C}$ e as amostras a imersas a $65^{\circ} \mathrm{Brix} / 50^{\circ} \mathrm{C}$ se diferiram das amostras imersas a $65^{\circ} \mathrm{Brix} / 70^{\circ} \mathrm{C}$ com relação à perda de massa e perda de água, a um nível de 5\% de probabilidade mostrando que amostras imersas em soluções com a mesma concentração foram influenciadas significativamente pela temperatura.

Tabela 4. Valores médios das variáveis: perda de massa, perda de água, incorporação de sólidos solúveis, taxa de encolhimento e capacidade de reidratação de cagaitas desidratadas osmoticamente.

\begin{tabular}{lccccc}
\hline Tratamentos & $\begin{array}{c}\text { Perda de } \\
\text { Massa }\end{array}$ & $\begin{array}{c}\text { Perda de } \\
\text { Água }\end{array}$ & $\begin{array}{c}\text { Incorporação de } \\
\text { Sólidos Solúveis }\end{array}$ & $\begin{array}{c}\text { Taxa de } \\
\text { Encolhimento }\end{array}$ & $\begin{array}{c}\text { Capacidade de } \\
\text { Reidratação }\end{array}$ \\
\hline $45^{\circ} \mathrm{Brix} / 50^{\circ} \mathrm{C}$ & $39,94 \mathrm{c}$ & $47,18 \mathrm{c}$ & $7,24 \mathrm{c}$ & $30,33 \mathrm{~d}$ & $13,08 \mathrm{a}$ \\
& & & $7,65 \mathrm{c}$ & $42,25 \mathrm{~b}$ & $9,42 \mathrm{c}$ \\
$45^{\circ} \mathrm{Brix} / 70^{\circ} \mathrm{C}$ & $64,99 \mathrm{a}$ & $72,64 \mathrm{a}$ & $7,65 \mathrm{c}$ & $53,48 \mathrm{c}$ & $11,21 \mathrm{~b}$ \\
& & & & & \\
$65^{\circ} \mathrm{Brix} / 50^{\circ} \mathrm{C}$ & $50,82 \mathrm{~b}$ & $60,83 \mathrm{~b}$ & $10,01 \mathrm{~b}$ & $58,11 \mathrm{a}$ & $7,04 \mathrm{~d}$ \\
& & & & &
\end{tabular}

Na coluna, as médias seguidas da mesma letra, não diferem entre si a $5 \%$ pelo teste de Tukey $(\mathrm{p} \leq 0.05)$.

Ao avaliar a desidratação osmótica de abacaxi, Beristain et al. [19] mencionaram que a taxa de desidratação osmótica é influenciada pela temperatura da solução. Resultado semelhante foi apresentado por Khoyi e Hesari [20] ao analisar a desidratação de damascos utilizando duas concentrações de sacarose. Os autores observaram que ao aumentar a temperatura em $30^{\circ} \mathrm{C}$ houve um aumento na perda de água dos damascos.

Diversas explicações são adotadas para descrever o efeito da temperatura, sendo que Khoyi e Hesari [20] atribuem esta maior perda de massa a uma redução da viscosidade da solução, atrelada a um aumento dos danos causados no tecido vegetal, levando ao aumento da difusão pelos tecidos.
No entanto, deve-se adotar durante a desidratação osmótica temperaturas que mantenham a qualidade dos vegetais [21], já que, a utilização de temperaturas mais elevadas causará modificações nutricionais e sensoriais no produto final [18].

Também foi possível observar que as amostras desidratadas em concentrações de $45^{\circ} \mathrm{Brix} / 50^{\circ} \mathrm{C}$ se diferiram significativamente das amostras desidratadas a $65^{\circ} \mathrm{Brix} / 50^{\circ} \mathrm{C}$ (Tabela 4), sugerindo que um aumento na concentração provocou um aumento na taxa de perda de água e de perda de massa das amostras.

Maior perda de massa e perda de água também foi encontrado por Moy et al. [22] ao desidratar frutas tropicais com maiores concentrações do agente osmótico. Ao elevar a 
concentração da solução osmótica de 55 para $65^{\circ}$ Brix, Lima et al. [23] (2004) encontraram maior perda de massa na desidratação osmótica de melão, confirmando o efeito da concentração do agente osmótico nas variáveis da desidratação.

Com relação à incorporação de sólidos solúveis podemos observar que amostras imersas nas soluções de $45^{\circ} \mathrm{Brix} / 50^{\circ} \mathrm{C}$ foram significativamente semelhantes às amostras imersas na solução de $45^{\circ} \mathrm{Brix} / 70^{\circ} \mathrm{C}$, que se diferiram das amostras desidratadas nas condições de $65^{\circ} \mathrm{Brix} / 50^{\circ} \mathrm{C}$ e $65^{\circ} \mathrm{Brix} / 70^{\circ} \mathrm{C}$ que apresentaram maiores valores de incorporação de sólidos solúveis (tabela 4). Este efeito da concentração também foi observado por Genina-Souto et al. [24] que ao avaliar batata-doce, registraram que houve um aumento na incorporação de sólidos solúveis nas amostras que foram imersas em maiores concentrações do agente osmótico.

Com relação à taxa de encolhimento podemos observar que as amostras de cagaita apresentaram diferença entre todos os tratamentos, sendo mais acentuado nas amostras desidratadas na temperatura mais elevada $\left(70^{\circ} \mathrm{C}\right)$ (Tabela 4).

Ao avaliar o coeficiente de encolhimento de tomates, Ribeiro [25] encontrou valores situados na faixa de 54,48 a $83,11 \%$, sendo que as maiores taxas de encolhimento foram observadas nas secagens com pré-tratamento osmótico. No entanto, Nsonzi \& Ramaswamy [26] observaram para o mirtilo uma taxa de encolhimento inferior a $10 \%$, sendo este muito inferior ao registrado no presente estudo.

A taxa de reidratação foi maior nos frutos de cagaita imersos na solução de $45^{\circ} \mathrm{Brix} / 50^{\circ} \mathrm{C}$ que se diferiu dos demais tratamentos (Tabela 4). Frutos desidratados nas condições de $65^{\circ} \mathrm{Brix} / 70^{\circ} \mathrm{C}$ (Tabela 4) apresentaram menor taxa de reidratação, o que pode ser atribuído a uma maior injúria da estrutura celular, visto que, a capacidade de reidratação está diretamente relacionada com o dano na parede celular.

A capacidade de reidratação está relacionada com a habilidade do vegetal desidratado absorver água e recuperar o volume perdido durante $o$ processo, sendo uma avaliação das lesões ocasionadas pelo método de desidratação [27].

Derlan et al. [28] determinaram a capacidade de reidratação de pimenta e afirmaram que os frutos secos a $40^{\circ} \mathrm{C}$ apresentaram maiores valores, o que pode ser atribuído a menores injúrias durante a desidratação.

As amostras de cagaita passas destinadas à avaliação sensorial foram aquelas que apresentaram a melhor avaliação da qualidade visual, realizada pelo condutor do experimento.

Três amostras de passas de cagaita $\left(45^{\circ} \mathrm{Brix} / 50^{\circ} \mathrm{C}, \quad 45^{\circ} \mathrm{Brix} / 70^{\circ} \mathrm{C}\right.$ e $\left.65^{\circ} \mathrm{Brix} / 50^{\circ} \mathrm{C}\right)$ foram encaminhadas à análise sensorial para a realização do teste de aceitação (Tabela 5).

Tabela 5. Valores médios de aceitação quanto aos atributos cor, sabor, aroma, impressão global e intenção de compra.

\begin{tabular}{cccccc} 
Tratamentos & Cor & Aroma & Sabor & $\begin{array}{c}\text { Impressão } \\
\text { Global }\end{array}$ & $\begin{array}{c}\text { Intenção de } \\
\text { Compra }\end{array}$ \\
\hline $45^{\circ} \mathrm{Brix} / 50^{\circ} \mathrm{C}$ & $6,82 \mathrm{a}$ & $6,02 \mathrm{a}$ & $6,03 \mathrm{a}$ & $6,22 \mathrm{a}$ & $3,01 \mathrm{a}$ \\
$45^{\circ} \mathrm{Brix} / 70^{\circ} \mathrm{C}$ & $5,83 \mathrm{~b}$ & $6,42 \mathrm{a}$ & $5,92 \mathrm{a}$ & $6,12 \mathrm{a}$ & $3,12 \mathrm{a}$ \\
$65^{\circ} \mathrm{Brix} / 50^{\circ} \mathrm{C}$ & $6,77 \mathrm{a}$ & $6,75 \mathrm{a}$ & $7,25 \mathrm{~b}$ & $6,02 \mathrm{a}$ & $4,68 \mathrm{~b}$
\end{tabular}

Na coluna, as médias seguidas da mesma letra, não diferem entre si a 5\% pelo teste de Tukey ( $\mathrm{p} \leq 0.05)$.

O atributo cor obteve maiores valores médios para as amostras imersas em soluções de $45^{\circ} \mathrm{Brix} / 50^{\circ} \mathrm{C}$ e $65^{\circ} \mathrm{Brix} / 50^{\circ} \mathrm{C}$ que foram significativamente iguais e diferentes das amostras imersas na solução de $45^{\circ} \mathrm{Brix} / 70^{\circ} \mathrm{C}$. Este fato pode ser atribuído a um leve escurecimento evidenciado nas amostras imersas na solução com maior temperatura. Para os atributos aroma e impressão global não houve diferença entre as três amostras.
Para o atributo sabor as maiores médias foram obtidas pela amostra $65^{\circ} \mathrm{Brix} / 50^{\circ} \mathrm{C}$, tal fato pode ser atribuído a um sabor mais adocicado apresentado pela amostra (relato dos provadores).

De acordo com os histogramas é possível afirmar que as maiores frequências de provadores atribuíram notas entre 6 a 9 para todos os atributos avaliados (Figura 1). 
Figura 01. Histogramas de frequência quanto à avaliação sensorial de cagaita passa.

Cor

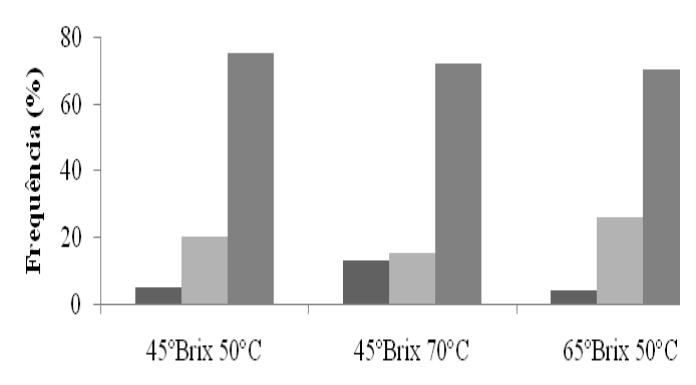

-Notas de 1 a 4 Notas 5 -Notas de 6a 9

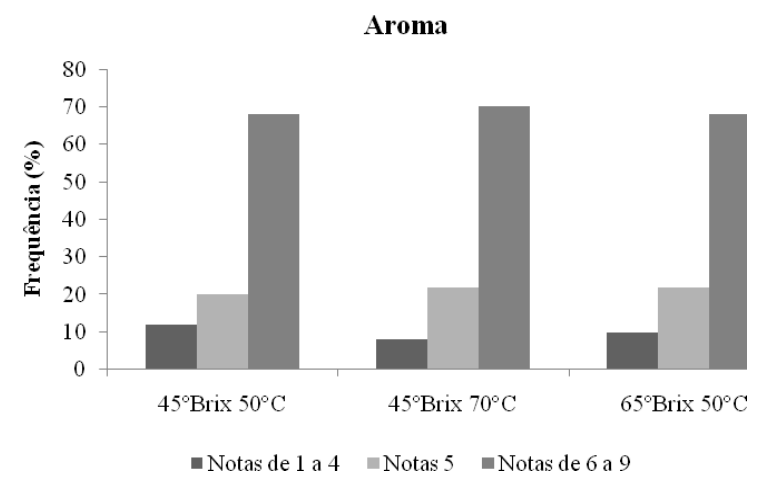

Ao avaliar a intenção de compra utilizando a escala hedônica de 5 pontos podemos observar que as amostras de passas de cagaita apresentaram uma boa intenção de compra com maiores frequências de provadores atribuindo notas entre certamente compraria e provavelmente compraria, sendo uma forma viável de agregação de valor ao fruto (Figura 02).

Figura 02. Histogramas de frequência quanto à intenção de compra de cagaita passa

\section{Intenção de Compra}

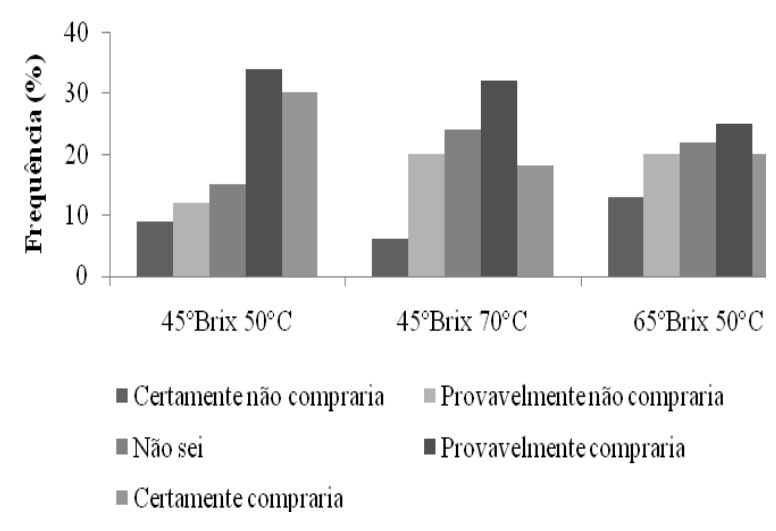

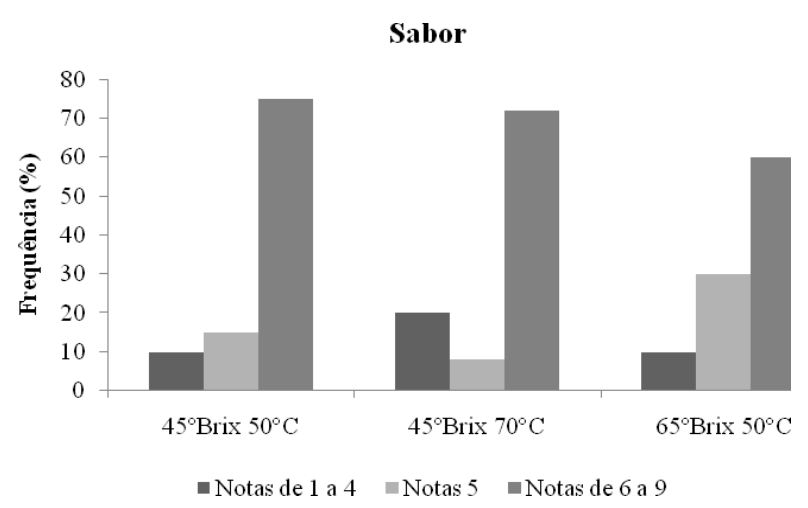

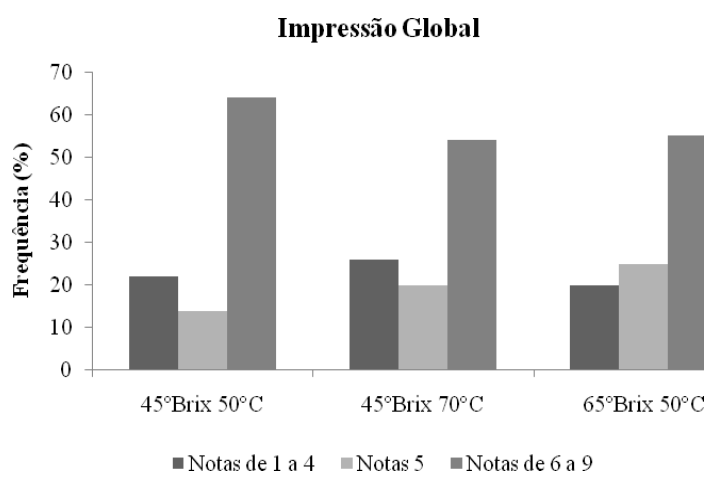

Após a realização da análise sensorial e definição do tratamento que apresentou maior intenção de compra procedeu-se a avaliação da composição centesimal. De acordo com a Tabela 3 observa-se que a cagaita passa apresentou um valor de $27,75 \%$ de umidade, $1,67 \%$ de proteínas, $0,82 \%$ de lipídios, 2,48\% de fibra, 0,29\% de cinzas e $66,89 \%$ de carboidratos. Elias et al. [29] (2008) ao avaliar as características nutricionais de caqui desidratado, observaram um aumento no teor de nutrientes entre $94 \%$ a $376 \%$, corroborando com os dados do presente estudo.

\section{CONCLUSÃO}

Os parâmetros da desidratação osmótica foram influenciados pelos fatores concentração do agente osmótico e temperatura da solução, sendo que as variáveis perda de massa, perda de água e incorporação de sólidos solúveis apresentaram valores mais elevados para os frutos que foram desidratados com concentração de $65^{\circ}$ Brix e a $70^{\circ} \mathrm{C}$.

A desidratação osmótica da cagaita para obtenção do fruto na forma de passa consiste numa alternativa interessante para agregação 


\section{CONTRIBUIÇÃO DOS AUTORES}

1. Planejamento, orientação, condução e avaliação do experimento, análises estatísticas e elaboração do artigo:

Caroline Dantas Mendes da Silva
$D^{\mathrm{a}}$. Caroline Roberta Freitas Pires

Dra $^{\mathrm{a}}$. Juliana Pinto de Lima

Anielli Souza Pereira

Clemilson Antônio da Silva

\section{REFERÊNCIAS}

[1]. ARAKAKI, A. H.; SCHEIDT, G. N.; PORTELLA, A. C.; ARRUDA, E. J.; COSTA, R. B. O baru (Dipteryx alata Vog.) como alternativa de sustentabilidade em área de fragmento florestal do Cerrado, no Mato Grosso do Sul. Interações, v.10, n.1, p.31-39, 2009. DOI:10.1590/S151870122009000100004

[2]. CARDOSO,M. L. ; MARTINO, H.S.D.; MOREIRA A.V.B.;RIBEIRO, S.M.R. ; PINHEIROSANT'ANA , H.M. Cagaita (Eugenia dysenrerica DC.) of the Cerrado of Minas Gerais, Brazil: Physical and chemical characterization, carotenoids and vitamins. Food Research International, v.44, n.7, p.21512154, 2011. DOI:10.1016/J.FOODRES.2011.03.005

[3]. TORREGGIANI, D.; BERTOLO, G. High-quality fruit and vegetable products using combined processes. In: FITO, P. et al. (Ed.). Osmotic dehydration and vacuum impregnation. Lancaster: Technomic Publishing Company, 2001. p. 3-9.

[4]. GUIMARÃES, M.G.; SILVA, M.S.; Valor nutricional e características químicas e físicas de frutos de murici-passa (Byrsonima verbascifolia). Ciência e Tecnologia de Alimentos, v.28, n.4, p.817-821, $2008 . \quad$ DOI:10.1590/S010120612011000100021

[5]. SANTOS, M.S. Impacto do processamento sobre as características físico-químicas, reológicas e funcionais de frutos da gabirobeira (Campomanesia xanthocarpa Berg). Tese. Universidade Federal do Paraná, 2011. 148p.

[6]. SOARES JÚNIOR, M.S.; REIS, R.C.; BASSINELLO, P.Z.; LACERDA, D.B.C.; KOAKUZU, S.N.; CALIARI, M. Qualidade de biscoitos formulados com diferentes teores de farinha dede casca de pequi. Pesquisa Agropecuária Tropical, v. 39, n. 2, p. 98-104.

[7]. LERICI, C.R.; PINNAVAIA, M.; DALLA ROSA, M.; BARTOLUCCI, L. Osmotic Dehydration of fruit: Influence of osmotic Agents on Drying Behavior and Product Quality. Journal of food Science, Chicago, v. 50, p. 1217-1219, 1985. DOI: $10.1111 / j .1365-2621.1985 . t b 10445 . x$

[8]. KARATHANOS, V.T.; KOSTAROPOULOS, A.E. Air-drying kinetics of osmotically dehydrated fruits. Drying Technology. v.13, n.5-7, p.1503-1521, 1995. DOI: $10.1080 / 07373939508917036$
[9]. BRANDÃO, M. C. C. MAIA, G.A.; LIMA, D.S.P.; PARENTE, E.J.S.A.; CAMPELLO, C.C.; NASSU, R.T.; TEREZINHA FEITOSA, T.; SOUSA, P.H.M. Análise físico-química, microbiológica e sensorial de frutos de manga submetidos à desidratação osmótico solar. Revista Brasileira de Fruticultura, v. 25 , n.1, p.38-41, 2003. DOI: $10.1590 / \mathrm{S} 0100-$ 29452003000100012

[10]. SOUSA, P. H. M.; MAIA, G.A.; FILHO, M.S.M.S.; FIGUEIREDO, R.W.F.; SOUZA, A.C.R. Goiabas desidratadas osmoticamente seguidas de secagem em estufa. Revista Brasileira de Fruticultura, v.25, n.3, p.414-416, 2003. DOI: 10.1590/S0100-29452003000300013

[11]. AOAC- ASSOCIATION OF OFFICIAL ANALYTICAL CHEMISTS. HORWITZ, W. Official methods of analysis of the Association Analytical Chemits. 17 ed Arlington: AOAC Inc., v.1 e v. 2, 2000.

[12]. ZOGZAS, N.P.; MAROULIS, Z.B.; KOURIS, D.M. Densities, skrinkage and porosity of some vegetables during ais drying. Drying Technology, $\begin{array}{llll}\text { v.12, n.7, } & \text { p.1653-1666, } & 1994 .\end{array}$ DOI:10.1080/07373939408962191

[13]. CORRÊA, J. L. G.; DEV, S.R.S.; GARIEPY, Y.; RAGHAVAN, G.S.V. Drying of pineapple by microwave-vacuum drying. Drying Technology, New York, v. 29, n.13, p. 1556-1561, Oct. 2011. DOI: 10.1080/07373937.2011.582558

[14]. CHAVES, J.B.P.; SPROESSER, R.L. Práticas de laboratório de análise sensorial de alimentos e bebidas. 1a ed. Viçosa: UFV; 2002.

[15]. FERREIRA, D.F. Análises estatísticas por meio do SISVAR para windows versão 4.0. In: REUNIÃO ANUAL DA REGIÃO BRASILEIRA DA SOCIEDADE INTERNACIONAL DE BIOMETRIA, 45, 2000, São Carlos, SP. Programa e Resumos... São Carlos: UFScar, 2000. p.235.

[16]. SILVA, A. S.; ALMEIDA, F.A.C.; SILVA, F.L.H.; DANTAS, H.J.; LIMA, E.E. Desidratação e efeito de pré-tratamentos no extrato seco do pimentão verde. Revista Brasileira de Produtos Agroindustriais, v. 10, n. 1, p. 27-34, 2008. DOI: $10.15871 / 1517-8595$

[17]. BORIN, I.; FRASCARELI, E.C.; MAURO, M.A.; KIMURA, M. Efeito do pré-tratamento osmótico com sacarose e cloreto de sódio sobre a secagem convectiva de abóbora. Ciência 
Tecnologia de Alimentos, v. 28, n. 1, p. 39-50, 2008. DOI:10.1590/S0101-20612008000100008

[18]. DHINGRA，D.; SINGH， J., PATIL， R.T., UPPAL, D.S. Osmotic dehydration of fruits and vegetables: A review. Journal of Food Science Technology, London, v.45, n.3, p.209-217, 2008. DOI: $1007 / \mathrm{s} 13197-012-0659-2$

[19]. BERISTAIN, C. I.; AZUARA, E., CORTES, R.; GARCIA, H. S. Mass transfer during osmotic dehydration of pineapple rings. International Journal of Food Science and Technology, v. 25, n. 1 , p.576-582, 1990. DOI: $10.1111 / \mathrm{j} .1365-$ 2621.1990.tb01117.x

[20]. KHOYI, M.R., HESARI, J. Osmotic dehydration kinetics of apricot using sucrose solution. Journal of Food Engineering, v.78, n.4, p.1355-1360, 2007. DOI:10.1016/J.JFOODENG.2006.01.007

[21]. ESCOBAR, M. P; GALINDO, F. G.; WADSÖ, L.; NÁJERA, J. R.; SJOHOLM, I. Effect of longterm storage and blanching pré-treatments on the osmotic kinectics of carrots (Daucus carota L. cv. Nerac). Journal of Food Engineering. v.81, n.1, p.313-317, 2007. DOI:10.1016/J.JFOODENG.2006.11.005

[22]. MOY, J.K.; LAU, B.H.; DOLLAR, A.M. Effects of sucrose and acid on osmotic-dehydration of tropical fruits. Journal of Food Processing and Preservation, Trumbull, v.2, n. 1, p.131-135, 1978. DOI: $10.1111 / \mathrm{j} .1745-4549.1978 . t b 00553 . \mathrm{x}$

[23]. LIMA, A. da S.; FIGUEIREDO, R. N. de; MAIA, G. A.; LIMA, J. R. e SOUZA, P. H. M. de. Estudo da Estabilidade de Melões Desidratados Obtidos por Desidratação Osmótica Seguida de Secagem Convencional. Revista Brasileira de Fruticultura, Jaboticabal, v. 26, n. 1, p. 107-109, 2004. DOI: $10.1590 / \mathrm{S} 0100-29452004000100029$
[24]. GENINA-SOUTO, P.; BARRERA-CORTES, J.; GUTIERREZ-LOPEZ， G.; NIETO, E.A. Temperature and concentration effects of osmotic media on profiles of sweet potato cubes. Drying Technology, v.19, n.3-4, p.547-558, 2001. DOI: 10.1081/DRT-100103933

[25]. RIBEIRO, R. C. Tomates semi desidratados obtidos por secagem micro-ondas convectiva precedida por desidratação osmótica. 2013. 87p. Dissertação (Mestrado em Ciência dos Alimentos) Departamento de Alimentos, Universidade Federal de Lavras, Lavras, 2013.

[26]. NSONZI, F.; RAMASWAMY, H. S. Osmotic dehydration kinetics of blueberries. Drying Technology, v. 16, n. 3, p. 725-741, 1998. DOI: $10.1080 / 07373939808917432$

[27]. KROKIDA, M. K.; MARINOS-KOURIS, D. Rehydration kinetics of dehydrated products. Journal of Food Engineering, v.57, n.1, p.1-7, 2003. DOI:10.1016/S0260-8774(02)00214-5

[28]. DERLAN, J. M.; SILVA, F. S.; PORTO, A. G. CINÉTICA DE REIDRATAÇÃO DE PIMENTA VARIEDADE BICO In: Congresso de Iniciação Científica, 5 ${ }^{\mathrm{a}}$. (JC), 2013, Cáceres/MT. Anais... Cáceres/MT: Pró-Reitoria de Pesquisa e PósGraduação - PRPPG, 2013. Vol. 8 (2013). Cód. 9230. ISSN ONLINE 2237-9258. CDROM 21787492.

[29]. ELIAS, N.F.; BERBERT, P.A.; MOLINA, M.A.B.; VIANA, A.P.; DIONELLO, R.G.; QUEIROZ, V.A.V.. Avaliação nutricional e sensorial de caqui cv Fuyu submetido à desidratação osmótica e secagem por convecção. Ciência Tecnologia de Alimentos, Campinas, v. 28, n.2, p.322-328, abr.-jun. 2008. DOI: 10.1590/S010120612008000200009. 